Louisiana State University

LSU Digital Commons

8-20-2004

\title{
Correlated X-ray and optical variability in V404 Cygni in quiescence
}

\author{
R. I. Hynes \\ The University of Texas at Austin \\ P. A. Charles \\ University of Southampton \\ M. R. Garcia \\ Harvard-Smithsonian Center for Astrophysics \\ E. L. Robinson \\ The University of Texas at Austin \\ J. Casares \\ Instituto Astrofisico de Canarias
}

See next page for additional authors

Follow this and additional works at: https://digitalcommons.Isu.edu/physics_astronomy_pubs

\section{Recommended Citation}

Hynes, R., Charles, P., Garcia, M., Robinson, E., Casares, J., Haswell, C., Kong, A., Rupen, M., Fender, R., Wagner, R., Gallo, E., Eves, B., Shahbaz, T., \& Zurita, C. (2004). Correlated X-ray and optical variability in V404 Cygni in quiescence. Astrophysical Journal, 611 (2 II) https://doi.org/10.1086/424005

This Article is brought to you for free and open access by the Department of Physics \& Astronomy at LSU Digital Commons. It has been accepted for inclusion in Faculty Publications by an authorized administrator of LSU Digital Commons. For more information, please contact ir@lsu.edu. 


\section{Authors}

R. I. Hynes, P. A. Charles, M. R. Garcia, E. L. Robinson, J. Casares, C. A. Haswell, A. K.H. Kong, M. Rupen, R. P. Fender, R. M. Wagner, E. Gallo, B. A.C. Eves, T. Shahbaz, and C. Zurita 


\section{UvA-DARE (Digital Academic Repository)}

\section{Correlated X-Ray and Optical Variability in V404 Cygni in Quiescence}

Hynes, R.I.; Charles, P.A.; Garcia, M.R.; Robinson, E.L.; Casares, J.; Haswell, C.A.; Kong, A.K.H.; Rupen, M.; Fender, R.P.; Wagner, R.M.; Gallo, E.; Eves, B.A.C.; Shahbaz, T.; Zurita, C.

DOI

10.1086/424005

Publication date 2004

Published in

Astrophysical Journal

Link to publication

Citation for published version (APA):

Hynes, R. I., Charles, P. A., Garcia, M. R., Robinson, E. L., Casares, J., Haswell, C. A., Kong, A. K. H., Rupen, M., Fender, R. P., Wagner, R. M., Gallo, E., Eves, B. A. C., Shahbaz, T., \& Zurita, C. (2004). Correlated X-Ray and Optical Variability in V404 Cygni in Quiescence.

Astrophysical Journal, 611, L125-L128. https://doi.org/10.1086/424005

\section{General rights}

It is not permitted to download or to forward/distribute the text or part of it without the consent of the author(s) and/or copyright holder(s), other than for strictly personal, individual use, unless the work is under an open content license (like Creative Commons).

\section{Disclaimer/Complaints regulations}

If you believe that digital publication of certain material infringes any of your rights or (privacy) interests, please let the Library know, stating your reasons. In case of a legitimate complaint, the Library will make the material inaccessible and/or remove it from the website. Please Ask the Library: https://uba.uva.nl/en/contact, or a letter to: Library of the University of Amsterdam, Secretariat, Singel 425, 1012 WP Amsterdam, The Netherlands. You will be contacted as soon as possible. 


\title{
CORRELATED X-RAY AND OPTICAL VARIABILITY IN V404 CYGNI IN QUIESCENCE
}

\author{
R. I. Hynes, ${ }^{1,2}$ P. A. Charles,${ }^{3}$ M. R. Garcia, ${ }^{4}$ E. L. Robinson,,${ }^{1}$ J. Casares, ${ }^{5}$ C. A. Haswell,${ }^{6}$ A. K. H. Kong, ${ }^{4}$ \\ M. Rupen, ${ }^{7}$ R. P. Fender, ${ }^{8}$ R. M. Wagner, ${ }^{9}$ E. Gallo, ${ }^{8}$ B. A. C. Eves, ${ }^{6}$ T. Shahbaz, ${ }^{5}$ And C. Zurita ${ }^{10}$ \\ Received 2004 May 14; accepted 2004 July 12; published 2004 July 21
}

\begin{abstract}
We report simultaneous X-ray and optical observations of V404 Cyg in quiescence. The X-ray flux varied dramatically by a factor of $\gtrsim 20$ during a $60 \mathrm{ks}$ observation. X-ray variations were well correlated with those in $\mathrm{H} \alpha$, although the latter include an approximately constant component as well. Correlations can also be seen with the optical continuum, although these are less clear. We see no large lag between X-ray and optical line variations; this implies they are causally connected on short timescales. As in previous observations, $\mathrm{H} \alpha$ flares exhibit a double-peaked profile suggesting emission distributed across the accretion disk. The peak separation is consistent with material extending outward to at least the circularization radius. The prompt response in the entire $\mathrm{H} \alpha$ line confirms that the variability is powered by X-ray (and/or EUV) irradiation.
\end{abstract}

Subject headings: accretion, accretion disks — binaries: close — stars: individual (V404 Cygni) — X-rays: binaries

\section{INTRODUCTION}

Accretion onto black holes is observed over a wide range of luminosities. While the upper end of the luminosity range, including bright $\mathrm{X}$-ray binaries and active galactic nuclei, is relatively accessible, quiescent or near-quiescent accretion is more difficult to study. There remain large uncertainties about the structure of the accretion flow in quiescence (see, e.g., Narayan et al. 2002), and it is possible that the energy output could be dominated by a jet rather than by the accretion flow itself (Fender et al. 2003). These accreting black holes emit across the electromagnetic spectrum from radio to X-rays, so multiwavelength studies can be used to disentangle different sources from different regions of the inflow, outflow, or jet and to establish causal connections between them.

This approach has had very little application for quiescent systems to date. Of the stellar mass black hole population, the most accessible quiescent object is V404 Cyg. V404 Cyg is known to vary in X-rays (Wagner et al. 1994; Kong et al. 2002), optical (Wagner et al. 1992; Casares et al. 1993; Pavlenko et al. 1996; Hynes et al. 2002; Zurita et al. 2003; Shahbaz et al. 2003), IR (Sanwal et al. 1996), and radio (Hjellming et al. 2000), but none of these studies were coordinated. Hynes et al. (2002) established that optical emission line variations are correlated with the optical continuum. They also found that

\footnotetext{
${ }^{1}$ McDonald Observatory and Department of Astronomy, University of Texas at Austin, 1 University Station C1400, Austin, TX 78712; rih@astro .as.utexas.edu.

${ }^{2}$ Hubble Fellow.

${ }^{3}$ School of Physics and Astronomy, University of Southampton, Southampton, SO17 1BJ, UK.

${ }^{4}$ Harvard-Smithsonian Center for Astrophysics, 60 Garden Street, MS-67, Cambridge, MA 02138.

${ }^{5}$ Instituto de Astrofísica de Canarias, Via Lactea, 38200 La Laguna, Tenerife, Spain.

${ }^{6}$ Department of Physics and Astronomy, Open University, Walton Hall, Milton Keynes, MK7 6AA, UK.

${ }^{7}$ National Radio Astronomy Observatory, Array Operations Center, 1003 Lopezville Road, Socorro, NM 87801.

${ }^{8}$ Astronomical Institute "Anton Pannekoek," University of Amsterdam, Kruislaan 403, 1098 SJ Amsterdam, Netherlands.

${ }^{9}$ Large Binocular Telescope Observatory, University of Arizona, 933 North Cherry Avenue, Tucson, AZ 85721.

${ }^{10}$ Centro de Astronomia e Astrofísica de Universidade de Lisboa, Observatório Astronómico de Lisboa, Tapada da Ajuda, 1349-018 Lisbon, Portugal.
}

the emission-line flares exhibited a double-peaked line profile, suggestive of emission distributed across the accretion disk (see, e.g., Horne \& Marsh 1986) rather than arising in localized regions. This was attributed to irradiation of the outer disk by the variable X-ray source, and hence it was predicted that the X-ray variations should be correlated with the optical. Such correlated variability, also attributed to irradiation, is commonly seen in X-ray-bright states in both neutron star systems and black holes (e.g., Grindlay et al. 1978; Petro et al. 1981; Hynes et al. 1998; and many other works) but had not been directly observed in quiescent systems. It is also usually only detected in the optical continuum yielding no kinematic information.

Here we report initial results from a coordinated, multiwavelength campaign to test this prediction. This included X-ray, nearUV, optical, and radio coverage, but this Letter discusses only the results from comparing X-ray data with optical spectroscopy. Future works will study the variability properties in more detail and examine the broadband spectral energy distribution.

\section{OBSERVATIONS}

\subsection{X-Ray Data}

Chandra observations on 2003 July 28/29 used the ACIS camera, in a single $61.2 \mathrm{ks}$ observation spanning binary phases 0.51-0.62. The source was positioned on the ACIS-S3 chip, and the $\frac{1}{8}$ subarray mode was used to reduce the frame time to $0.4 \mathrm{~s}$ and hence ensure that pileup was negligible. Data analysis was performed with CIAO, version 3.0. Source events were extracted from a $3^{\prime \prime}$ radius aperture, with events with energies of $0.3-7.0 \mathrm{keV}$ retained; a total of 1941 such events were recorded, corresponding to an average count rate of 0.03 counts $\mathrm{s}^{-1}$, a factor of 5 lower than the previous observation (Garcia et al. 2001; Kong et al. 2002). The background count rate was approximately constant, produced $\sim 4$ counts in the source aperture, and was neglected for subsequent analysis. The spectrum will not be discussed here but was very similar to that seen by Kong et al. (2002). Based on this spectrum, and assuming a distance of $3.5 \mathrm{kpc}$, we estimate that 1 count $\mathrm{s}^{-1}$ corresponds to an unabsorbed $0.3-7.0 \mathrm{keV}$ luminosity of $3.2 \times 10^{34} \mathrm{ergs} \mathrm{\textrm {s } ^ { - 1 }}$. 


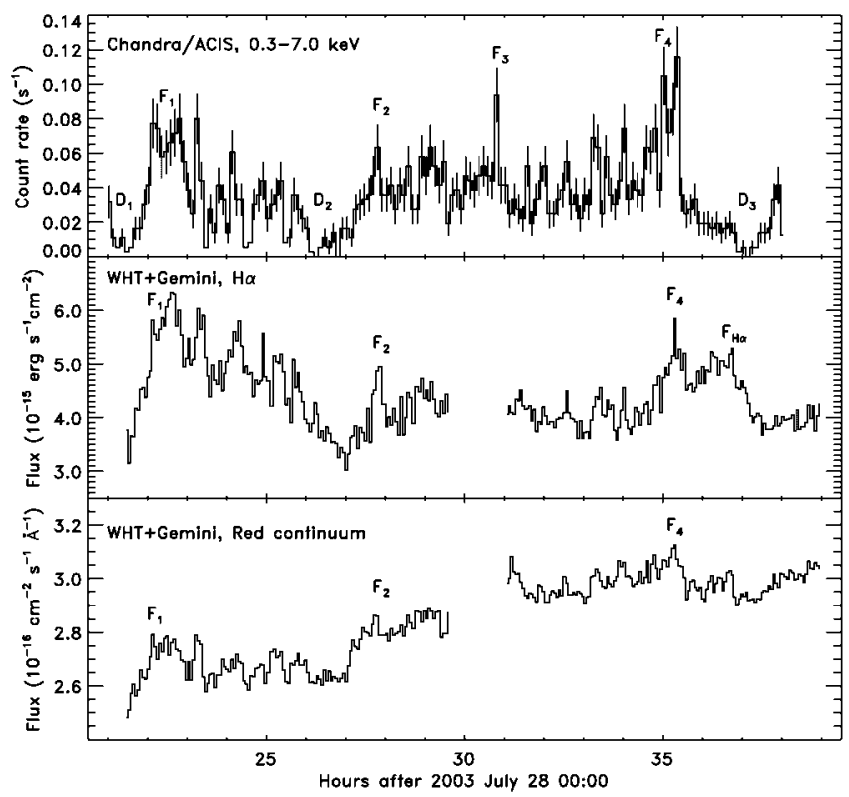

FIg. 1.-Simultaneous X-ray and optical light curves. Annotations refer to pronounced dips in the X-ray light curve $\left(\mathrm{D}_{1}-\mathrm{D}_{3}\right)$, selected flares $\left(\mathrm{F}_{1}-\mathrm{F}_{4}\right)$, and a flare seen in $\mathrm{H} \alpha$ that is not present in $\mathrm{X}$-rays $\left(\mathrm{F}_{\mathrm{H} \alpha}\right)$. X-ray data is plotted in $400 \mathrm{~s}$ bins, and approximately 1 count s $\mathrm{s}^{-1}$ corresponds to an unabsorbed $0.3-$ $7.0 \mathrm{keV}$ luminosity of $3.2 \times 10^{34} \mathrm{ergs} \mathrm{s}^{-1}$. The first half of the optical coverage is from the WHT, the second half from Gemini. WHT corresponds to individual $200 \mathrm{~s}$ exposures, and Gemini shows $40 \mathrm{~s}$ exposures binned by a factor of 4 to match the WHT resolution. The continuum bandpass is $6300-6500 \AA$ plus $6620-6820 \AA$. The overall continuum rise is the ellipsoidal modulation of the companion star. Error bars are omitted from the optical data for clarity but are much smaller than those on the $\mathrm{X}$-ray data.

\subsection{Optical Spectrophotometry}

The first half of our time-resolved optical spectrophotometry was obtained with the ISIS dual-arm spectrograph on the William Herschel Telescope (WHT). To maximize efficiency and minimize readout time and noise, we used the single red-arm mode with the R316R grating and MARCONI2 CCD. Exposure times were $200 \mathrm{~s}$, with $\sim 17 \mathrm{~s}$ dead time between exposures. A $4 "$ slit was used to maximize photometric accuracy, so our spectral resolution was determined by the seeing (median $\sim 1 " 3$ ) and was typically $\sim 5.5 \AA\left(250 \mathrm{~km} \mathrm{~s}^{-1}\right)$. Bias correction and flat-fielding were performed using standard IRAF ${ }^{11}$ techniques. The slit was aligned to cover the same comparison star as used for our previous observations of the target, and spectra of both of these stars, and the nearby blended star, were extracted with the same techniques previously described (Hynes 2002; Hynes et al. 2002). Wavelength calibration was performed relative to a single observation of a $\mathrm{CuNe} / \mathrm{CuAr}$ lamp. Time-dependent variations in the wavelength calibration were corrected using telluric absorption features. The on-slit comparison star was calibrated relative to Kopff 27 (Stone 1977), and all spectra of V404 Cyg were calibrated relative to this on-slit comparison.

The second half of our optical coverage was provided by the Gemini Multi-Object Spectrograph on Gemini-North. We used the R831 grating and standard EEV CCDs. Exposure times were $40 \mathrm{~s}$, and binning and windowing were used to reduce the dead time between exposures to $12 \mathrm{~s}$. A $5^{\prime \prime}$ slit with $1{ }^{\prime \prime} 1$

\footnotetext{
${ }^{11}$ IRAF is distributed by the National Optical Astronomy Observatory, which is operated by the Association of Universities for Research in Astronomy (AURA), Inc., under cooperative agreement with the National Science Foundation.
}

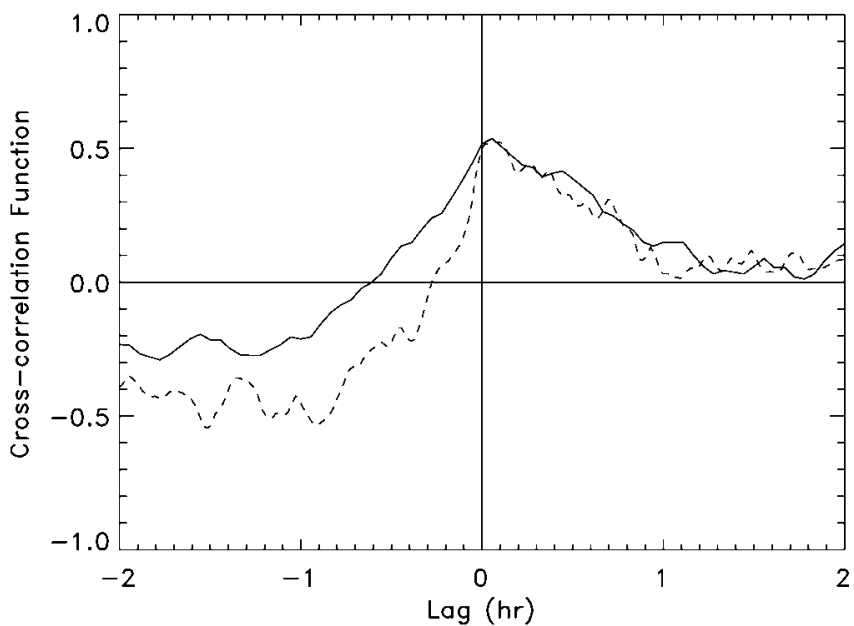

FIG. 2.-X-ray vs. $\mathrm{H} \alpha$ cross-correlation functions. The solid line is derived from the first (WHT) segment. The dashed line corresponds to the Gemini segment, terminated after flare $\mathrm{F}_{4}$ to avoid contamination by the $\mathrm{H} \alpha$ flare $\mathrm{F}_{\mathrm{H} \alpha}$. Positive lags correspond to the optical lagging the $\mathrm{X}$-rays.

median seeing resulted in a spectral resolution of $5.0 \AA$ (230 $\mathrm{km} \mathrm{s}^{-1}$ ). Data reduction, spectral extraction, and wavelength and flux calibration were performed in the same way as for the WHT data. Wavelength calibration used a CuAr lamp, and flux calibration was performed relative to the same on-slit comparison star as used for the WHT observations.

\section{LIGHT CURVES}

$\mathrm{X}$-ray and optical light curves are shown in Figure 1. Dramatic X-ray variability is clearly present with a dynamic range of greater than a factor of 20 , comparable to that seen by Wagner et al. (1994) on longer timescales. The lowest count rates seen in three dips $\left(D_{1}-D_{3}\right)$ correspond to a luminosity of $\$ 10^{32} \mathrm{ergs} \mathrm{s}^{-1}$, which is comparable to the $\mathrm{X}$-ray luminosities of other quiescent black holes (Garcia et al. 2001). Clear correlations are seen between the X-ray flux and that in both $\mathrm{H} \alpha$ and the optical continuum in overall trends and in the distinct flares (e.g., $F_{1}, F_{2}$, and $F_{4}$ ). One unusually fast X-ray flare occurred $\left(\mathrm{F}_{3}\right)$, lasting for $\$ 200 \mathrm{~s}$ and reaching a peak count rate (unresolved in Fig. 1) in excess of 0.25 counts $\mathrm{s}^{-1}$. While $\mathrm{H} \alpha$ generally tracks the X-rays rather well, one $\mathrm{H} \alpha$ flare (marked $\mathrm{F}_{\mathrm{H} \alpha}$ ) occurs when the X-rays are low and declining; this appears unrelated to X-ray behavior. The continuum apparently tracks the X-ray behavior less well than $\mathrm{H} \alpha$.

To measure the lag between the X-ray and $\mathrm{H} \alpha$ light curves, we calculated interpolation cross-correlation functions (CCFs; Gaskell \& Peterson 1987; White \& Peterson 1994). These are shown in Figure 2. The WHT and Gemini data were used unbinned and cross-correlated against X-ray light curves with 200 and $50 \mathrm{~s}$ time resolution, respectively. These CCFs exhibit a very similar structure to the line versus continuum CCFs presented by Hynes et al. (2002). There is clearly no large lag in the line response to within a few hundred seconds. Viscous, thermal, and even dynamical timescales in the line-formation region are likely to be greater than this, so coupling on these timescales is inconsistent with the observations. A lag corresponding to the light-travel time across the disk ( $\lesssim 40 \mathrm{~s}$ ) is possible, and indeed the data suggest a positive lag larger than this. We do not claim detection of a nonzero lag without more detailed examination of the data, however, and defer this to a later work. If the lag is larger than expected from light-travel 


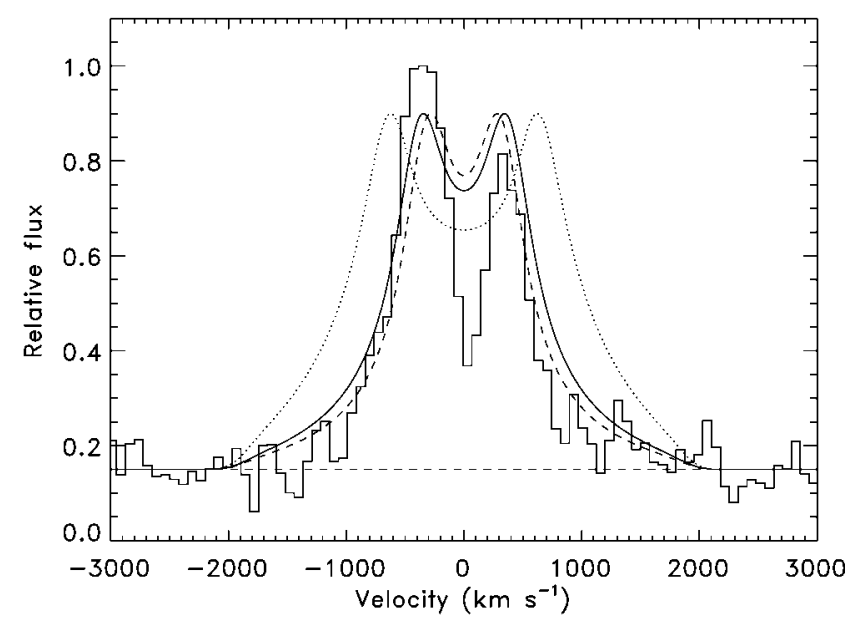

FIG. 3.-Optical spectrum near $\mathrm{H} \alpha$ of $\mathrm{F}_{1}$. The histogram shows the data. Note that the continuum flare spectrum has not been subtracted; this is approximated by the dashed line. The smooth lines show representative optically thin line profiles. The solid line corresponds to an outer edge at $R_{\text {circ }}$; dashed lines correspond to a tidally truncated disk and dotted lines to a disk extending to $R_{\text {circ }} / 3$.

times, then one explanation might be a finite reprocessing time, as this might be rather large for the cool atmospheres expected in the disk in quiescence (Cominsky et al. 1987; McGowan et al. 2003).

\section{LINE PROFILES}

We show in Figure 3 a section of the spectrum of flare $F_{1}$, calculated as the difference between the spectra during a trough at $21.5 \mathrm{hr}$ and the peak at $22.5 \mathrm{hr}$. The $\mathrm{H} \alpha$ line clearly exhibits double-peaked enhancements during the flare, similar to the difference profiles obtained by Hynes et al. (2002). This indicates that the line response is dominated by the accretion disk rather than by the companion star or stream-impact point. We have shown a representative optically thin line profile for comparison, smoothed to match the spectral resolution. The only characteristics we have attempted to reproduce are the flux and separation of the peaks. The other parameters assumed are an inner disk radius $10^{4} R_{\mathrm{Sch}}$, black hole mass $12 M_{\odot}$, and inclination $56^{\circ}$ (Shahbaz et al. 1994). We assume the emissionline surface brightness varies as $R^{-1.7}$ (Horne \& Marsh 1986). The peak separation is well fitted with a disk with outer edge at the circularization radius, estimated to be $R_{\text {circ }}=(9.2 \pm$ $0.4) \times 10^{11} \mathrm{~cm}$ with parameters from Casares \& Charles (1994) and Shahbaz et al. (1994). A significantly smaller outer disk radius, as shown in Figure 3, is not consistent with the data, although it is possible for the illuminated region to extend to the tidal truncation radius, $\sim 1.3 \times 10^{12} \mathrm{~cm}$. This profile is clearly inadequate in other ways; it does not reproduce the wings or central minimum well, and the asymmetry of the peaks is unaccounted for. The key result for our purposes, the outer radius of the emitting region, is largely insensitive to modifications to reproduce the shapes of the wings and the core, and the asymmetry, and so is a robust result for this flare. The other flares are shorter and/or weaker, so the flare line profile is less well constrained.

Material outside of $R_{\text {circ }}$ is expected to have a dynamical timescale of $\geq 6 \mathrm{hr}$. This is much longer than most of the events in the light curve, in particular the early rise to $F_{1}$ from which the flare line profile was derived, and is also much longer than any lag between the X-ray and $\mathrm{H} \alpha$ light curves (Fig. 2). The

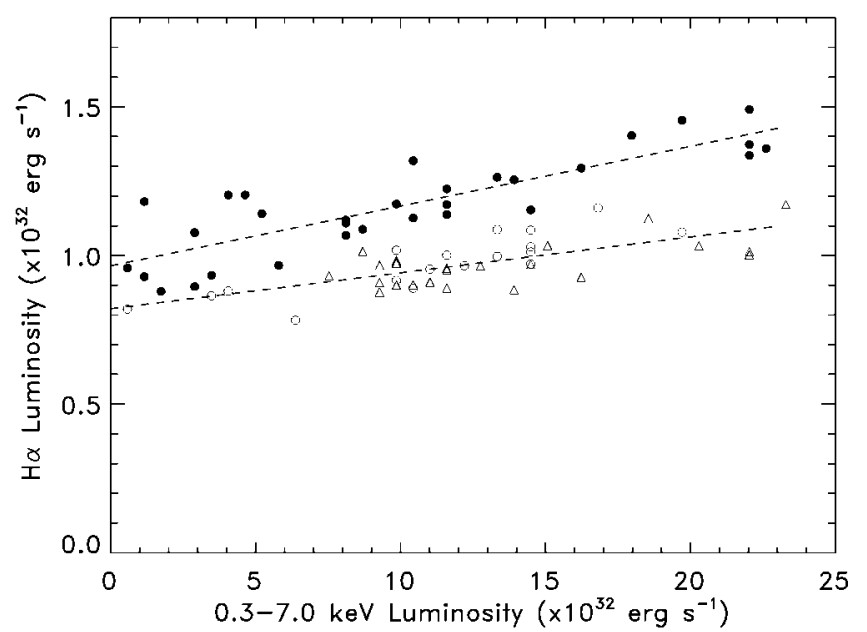

FIG. 4.-Relationship between X-ray and optical luminosities. Conversion to luminosity assumes a distance of $3.5 \mathrm{kpc}$, constant X-ray spectral shape, absorption column $8 \times 10^{21} \mathrm{~cm}^{-2}$ (estimated from a power-law fit to the $0.3-$ $7.0 \mathrm{keV}$ spectrum), and optical extinction $A_{V}=4.0$. The X-ray emission is assumed to be isotropic. $\mathrm{H} \alpha$ emission has been corrected under the assumption that it originates from a disk at an inclination of $56^{\circ}$. Filled circles correspond to the interval $D_{1}-D_{2}$; open symbols correspond to $D_{2}$ to the end of $F_{4}$. The period after $\mathrm{F}_{4}$ was excluded because of the presence of the $\mathrm{H} \alpha$ flare $\mathrm{F}_{\mathrm{H} \alpha}$. Circles indicate WHT data; triangles are from Gemini. Dashed lines indicate linear fits.

only plausible way to couple the whole line profile to the $\mathrm{X}$ ray variations is therefore through irradiation, as the light-travel time to $R_{\text {circ }}, \sim 30 \mathrm{~s}$, is easily short enough. We note that the dynamical timescale at $R_{\text {circ }}$ is actually comparable to the $6 \mathrm{hr}$ quasi periodicity reported in both photometry and line behavior by Casares et al. (1993) and Pavlenko et al. (1996).

\section{FLARE ENERGETICS}

Figure 4 shows the relationship between X-ray and $\mathrm{H} \alpha$ luminosities. There is no evidence for an uncorrelated X-ray component; the correlation extends to almost zero X-ray flux, and the light curves show no X-ray features that are not reproduced by $\mathrm{H} \alpha$ (except possibly at the very end of the light curve). There is, however, an approximately constant component to the $\mathrm{H} \alpha$ emission, which varies only slowly.

From the linear fits shown in Figure 4, the variable component of $\mathrm{H} \alpha$ luminosity corresponds to $2.0 \%$ of the X-ray luminosity in the first segment and $1.2 \%$ in the second. Neither of the quantities compared are bolometric luminosities. The observed X-ray luminosity (assumed to be isotropic) is a lower limit on the bolometric irradiating luminosity, which also includes EUV and $\gamma$-ray emission. $\mathrm{H} \alpha$ provides a lower limit on the reprocessed luminosity. More detailed modeling will be needed to estimate these bolometric corrections. If we consider only irradiation that can ionize neutral hydrogen (i.e., above $13.6 \mathrm{eV}$ ), and below $100 \mathrm{keV}$, then this is unlikely to exceed the X-rays by more than a factor of a few; for example, it is about a factor of 3 for a pure power-law spectrum (photon index $\Gamma=1.8$ ) and a factor of 5 for model 1 of Narayan et al. (1997). Such a low-energy cutoff is somewhat arbitrary but is also motivated by the large uncertainty concerning optical synchrotron emission in the models of Narayan et al. (1997). This contributes most of the truly bolometric luminosity but is much weaker in more recent models (e.g., Quataert \& Narayan 1999; Ball et al. 2001). $\mathrm{H} \alpha$ will not exceed $20 \%-30 \%$ of the repro- 
cessed luminosity, where the limit corresponds to case B recombination (Osterbrock 1989); it is likely to be substantially less than this. Thus, the reprocessed fraction is likely to be at least a few percent, although this is not a solid, model-independent constraint. For a thin disk and isotropic irradiation, the fraction intercepted is approximately $H / R$, so the lower limit is plausible for a central compact $\mathrm{X}$-ray source irradiating a thin disk $(H / R \geqslant 0.02)$. However, there is also a significant component of the optical continuum that is correlated with $\mathrm{X}$-rays (Figs. 1 and 3 ). If this also originates in reprocessed $\mathrm{X}$-rays, then the reprocessed fraction would be larger, as the optical continuum flux exceeds that in $\mathrm{H} \alpha$ by a factor much larger than plausible bolometric corrections to the irradiating flux. This case would then favor an elevated or vertically extended X-ray emission geometry that allows more efficient illumination of the disk. The variable optical continuum component could alternatively be dominated by synchrotron emission (e.g., Kanbach et al. 2001; Hynes et al. 2003).

\section{CONCLUSIONS}

We have established that optical and X-ray variations in V404 Cyg in quiescence are fairly well correlated. All X-ray variability (accounting for essentially all of the observed Xray flux) is mirrored well by $\mathrm{H} \alpha$ and to a lesser extent by the optical continuum. There is clearly another component of $\mathrm{H} \alpha$ emission, which exhibits rarer, or less pronounced variations, but is not completely constant. The correlated $\mathrm{H} \alpha$ component exhibits double-peaked line profiles indicating emission from a disk. The peak separation implies that the outer edge of the emitting region is at or outside the circularization radius. The timescales of the flares, significantly less than the dynamical timescale at the circularization radius, suggest that the X-ray$\mathrm{H} \alpha$ connection is mediated by irradiation of the accretion disk. The correlated $\mathrm{H} \alpha$ has a luminosity of approximately $1 \%-2 \%$ of the $0.3-7.0 \mathrm{keV} \mathrm{X}$-ray luminosity, which is consistent with an irradiation model. Our results therefore demonstrate that Xray/EUV irradiation has a measurable effect even in quiescent black hole X-ray transients and that optical observations can be used to perform an indirect study of X-ray (i.e., inner disk) variability, at least for V404 Cyg.

R. I. H. is supported by NASA through Hubble Fellowship grant HF-01150.01-A awarded by the Space Telescope Science Institute, which is operated by AURA, for NASA, under contract NAS 5-26555. Chandra observations were supported by NASA grant GO3-4044X. The WHT is operated on La Palma by the Isaac Newton Group in the Spanish Observatorio del Roque de los Muchachos of the Instituto de Astrofísica de Canarias. The Gemini Observatory is operated by AURA, under a cooperative agreement with the NSF on behalf of the Gemini partnership: NSF (US), PPARC (UK), NRC (Canada), CONICYT (Chile), ARC (Australia), CNPq (Brazil), and CONICET (Argentina). This work has also made use of the NASA ADS abstract service.

\section{REFERENCES}

Ball, G. H., Narayan, R., \& Quataert, E. 2001, ApJ, 552, 221

Casares, J., \& Charles, P. A. 1994, MNRAS, 271, L5

Casares, J., Charles, P. A., Naylor, T., \& Pavlenko, E. P. 1993, MNRAS, 265, 834

Cominsky, L. R., London, R. A., \& Klein, R. I. 1987, ApJ, 315, 162

Fender, R. P., Gallo, E., \& Jonker, P. G. 2003, MNRAS, 343, L99

Garcia, M. R., McClintock, J. E., Narayan, R., Callanan, P., Barret, D., \& Murray, S. S. 2001, ApJ, 553, L47

Gaskell, C. M., \& Peterson, B. M. 1987, ApJS, 65, 1

Grindlay, J. E., McClintock, J. E., Canizares, C. R., Cominsky, L., Li, F. K., Lewin, W. H. G., \& van Paradijs, J. 1978, Nature, 274, 567

Hjellming, R. M., Rupen, M. P., Mioduszewski, A. J., \& Narayan, R. 2000, Astron. Telegram 54

Horne, K., \& Marsh, T. R. 1986, MNRAS, 218, 761

Hynes, R. I. 2002, A\&A, 382, 752

Hynes, R. I., O'Brien, K., Horne, K., Chen, W., \& Haswell, C. A. 1998, MNRAS, 299, L37

Hynes, R. I., Zurita, C., Haswell, C. A., Casares, J., Charles, P. A., Pavlenko, E. P., Shugarov, S. Y., \& Lott, D. A. 2002, MNRAS, 330, 1009

Hynes, R. I., et al. 2003, MNRAS, 345, 292

Kanbach, G., Straubmeier, C., Spruit, H. C., \& Belloni, T. 2001, Nature, 414, 180

Kong, A. K. H., McClintock, J. E., Garcia, M. R., Murray, S. S., \& Barret, D. 2002, ApJ, 570, 277

McGowan, K. E., Charles, P. A., O'Donoghue, D., \& Smale, A. P. 2003, MNRAS, 345, 1039
Narayan, R., Barret, D., \& McClintock, J. E. 1997, ApJ, 482, 448

Narayan, R., Garcia, M. R., \& McClintock, J. E. 2002, in The Ninth Marcel Grossmann Meeting, ed. V. G. Gurzadyan, R. T. Jantzen, \& R. Ruffini (Singapore: World Scientific), 405

Osterbrock, D. E. 1989, Astrophysics of Gaseous Nebulae and Active Galactic Nuclei (Mill Valley: University Science Books)

Pavlenko, E. P., Martin, A. C., Casares, J., Charles, P. A., \& Ketsaris, N. A. 1996, MNRAS, 281, 1094

Petro, L. D., Bradt, H. V., Kelley, R. L., Horne, K., \& Gomer, R. 1981, ApJ, 251, L7

Quataert, E., \& Narayan, R. 1999, ApJ, 520, 298

Sanwal, D., Robinson, E. L., Zhang, E., Colome, C., Harvey, P. M., Ramseyer, T. F., Hellier, C., \& Wood, J. H. 1996, ApJ, 460, 437

Shahbaz, T., Dhillon, V. S., Marsh, T. R., Zurita, C., Haswell, C. A., Charles, P. A., Hynes, R. I., \& Casares, J. 2003, MNRAS, 346, 1116

Shahbaz, T., Ringwald, F. A., Bunn, J. C., Naylor, T., Charles, P. A., \& Casares, J. 1994, MNRAS, 271, L10

Stone, R. P. S. 1977, ApJ, 218, 767

Wagner, R. M., Kreidl, T. J., Howell, S. B., \& Starrfield, S. G. 1992, ApJ, 401, L97

Wagner, R. M., Starrfield, S. G., Hjellming, R. M., Howell, S. B., \& Kreidl, T. J. 1994, ApJ, 429, L25

White, R. J., \& Peterson, B. M. 1994, PASP, 106, 879

Zurita, C., Casares, J., \& Shahbaz, T. 2003, ApJ, 582, 369 